NEWS

\title{
'Seek, test and treat' slows HIV
}

\section{Studies in several nations show that treating people before they fall ill can curb the spread of disease.}

Treating HIV infection aggressively before symptoms appear could help to control the spread of the disease, according to data presented at a retroviral conference last week. Independent studies in Canada, the United States and Africa support the strategy in both the developed and developing world. However, the studies are not definitive and some scientists argue that improperly expanding treatment could cause problems.

Evidence in favour of 'treatment as prevention' has been building for more than a decade. Treating pregnant women who are HIV-positive, for instance, decreases disease transmission to their babies by lowering virus levels in the mother ${ }^{1}$. Modellers with the World Health Organization last year predicted that HIV could be almost eliminated within 50 years by finding and treating every HIV-infected person immediately - rather than waiting for the disease to advance to the point at which treatment is recommended by current guidelines ${ }^{2}$.

At the annual Conference on Retroviruses and Opportunistic Infections in San Francisco, California, on 16-19 February, Deborah Donnell of the Fred Hutchinson Cancer Research Center in Seattle, Washington, bolstered the 'seek, test and treat' strategy with data from a study of 3,408 couples from seven African nations in which only one partner had HIV. During the study, HIV-positive individuals who were receiving treatment almost never passed the virus on to their partner, whereas many untreated individuals did.

In another study, Moupali Das of the San Francisco Department of Public Health reported

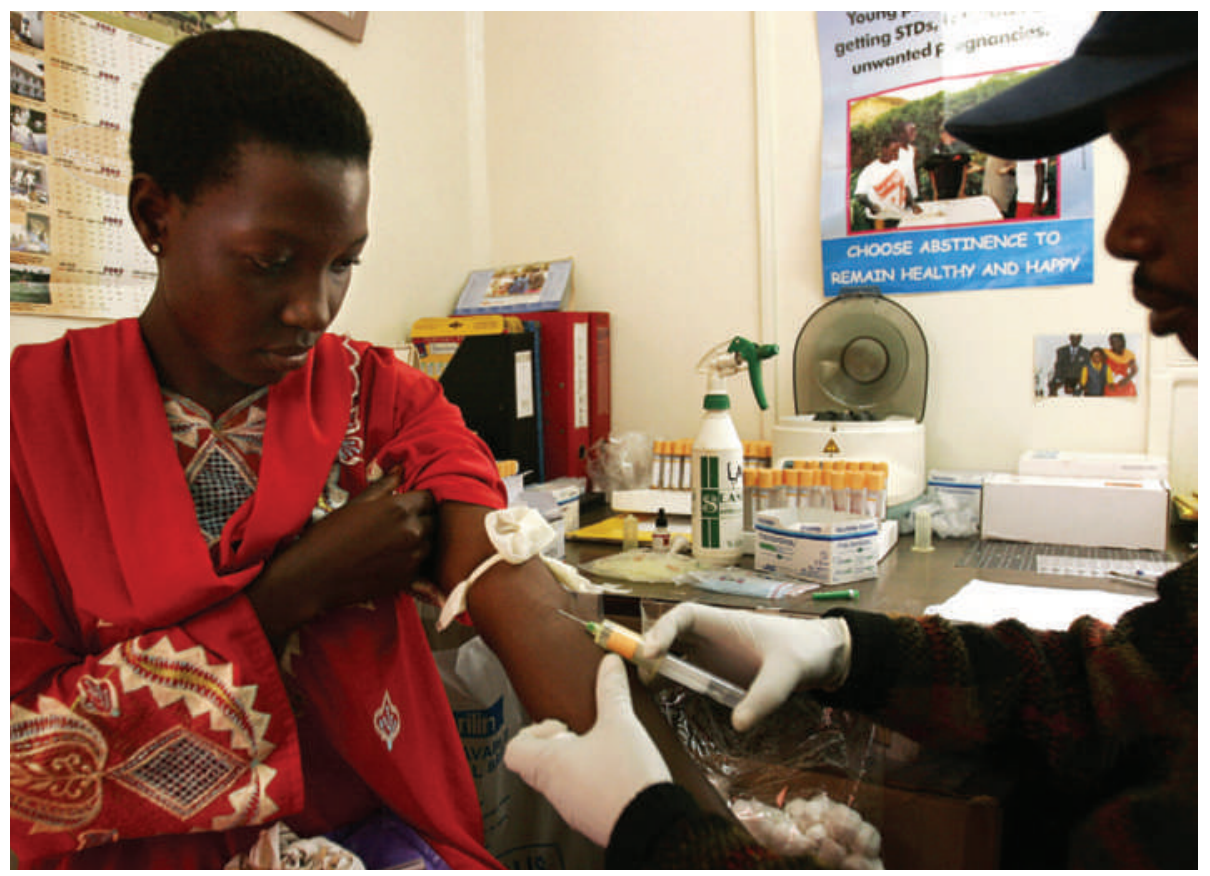

Testing whole populations for HIV and treating those infected may slow the spread of the disease.

the epidemic is clear at a population level, and we would like to see the seek, test and treat strategy endorsed throughout the developed and developing world," Montaner says.

However, these studies do not prove that treatment itself caused the declines in new infections because there were many confounding factors beyond the investigators control. And other data presented at the meeting introduced a cautionary note about the strategy. that as 'community viral load' - the amount of virus in the blood of all HIV-infected individuals tested in San Francisco - declined from 2005 to 2008 because of drug treatment and increased awareness, the number of new infections in the city also dropped. Julio Montaner, director of the British
"Rather than an all-or-nothing phenomenon, let test and treat be part of a more aggressive prevention armamentarium."
Sally Blower of the University of California, Los Angeles, argued that scarce resources in developing countries would be better spent reaching the more than 9 million people who already need treatment and are not getting it. And she reported on model results suggesting that if therapies were offered to Columbia Centre for Excellence in HIV/AIDS, described similar results last year for a study of intravenous drug users in Vancouver ${ }^{3}$.

At the conference, he reported that when treatment was expanded to intravenous drug users with HIV throughout British Columbia, new HIV diagnoses in that group dropped by around $50 \%$.

"The fact that more treatment would curb almost everyone in South Africa who needed them, drug-resistant strains could surge until they comprised up to $20 \%$ of circulating HIV strains.

Yet in Montaner's study, drug resistance actually dropped across the province when treatment was extended to all intravenous drug users.

Clinical trials of the seek, test and treat strategy will begin this year in New York and Washington DC, and others are planned by French and British agencies. A randomized trial already under way in nine countries is testing whether early treatment leads to durable prevention of transmission within couples. That study will not report results for several years.

Even if the most optimistic models are not correct, more aggressive efforts to expand treatment to everyone will probably dent the spread of the epidemic, says Anthony Fauci, director of the US National Institute of Allergy and Infectious Diseases in Bethesda, Maryland.

"Rather than saying it's an all-or-nothing phenomenon - that we're going to eliminate the epidemic without anything else but test and treat - what I argue for is, why don't we let test and treat be part of a more aggressive prevention armamentarium," Fauci says. "I would be satisfied with the epidemic if not disappearing, then at least declining, and I see seek, test and treat as one of several tools in the tool kit that will get us there."

Erika Check Hayden

\footnotetext{
1. De Cock, K. M. et al. J. Am. Med. Assoc. 283, 1175-1182 (2000).

2. Granich, R. M. et al. Lancet 373, 48-57 (2009).

3. Wood, E. et al. Br. Med. J. 338, b1649 (2009).
} 